\title{
PHASE UNWRAPPING CORRECTION WITH DUAL-BASELINE DATA FOR THE TANDEM-X MISSION
}

\author{
Marie Lachaise ${ }^{1}$, Thomas Fritz ${ }^{1}$, Ulrich Balss ${ }^{1}$, Richard Bamler ${ }^{1,2}$, Michael Eineder ${ }^{1}$ \\ ${ }^{1}$ Remote Sensing Technology Institute IMF \\ German Aerospace Center DLR \\ 82230 Wessling, Germany \\ ${ }^{2}$ Chair of Remote Sensing Technology, Technische Universität München \\ 80333 Munich, Germany
}

\begin{abstract}
The operational dual-baseline phase unwrapping algorithm for the TanDEM-X mission is based on a combination of separate single-baseline phase unwrapping and a correction procedure of different levels of complexity. It benefits from all the information available from the two TanDEM-X acquisitions by computing a differential interferogram to obtain a more reliable unwrapped phase. This may still be prone to phase unwrapping errors, but these can be mitigated using a stereo-radargrammetric measurement. Hence, the resulting dual-baseline phase unwrapping algorithm outperforms a single-baseline one.
\end{abstract}

Index Terms - phase unwrapping, TanDEM-X, differential interferogram, stereo-radargrammetry

\section{INTRODUCTION}

The TanDEM-X Mission, started on June $21^{\text {st }}$, 2010, has the primary objective to generate a global Digital Elevation Model (DEM) with an unprecedented accuracy [1]. This requires high coherence and reliable phase unwrapping. To achieve this accuracy, the whole land mass will be mapped twice: the first coverage has been done in 2011, and the second is being acquired in 2012. Over the second year, two interferograms with the same geometry (same orbital track, thus same incidence and look angles) but with different baselines and with shifted beams are available. Previously, we presented an approach to unwrap simultaneously both phases using Maximum A Posteriori estimation [2]. However, it turned out not to be robust enough because of noise and temporal decorrelation between the two TanDEM$\mathrm{X}$ acquisitions and this approach degenerated to lead to unacceptable error amplification. Looking for local height discrepancies and correcting them afterwards is a better strategy and is well-suited for dual-baseline phase unwrapping for the TanDEM-X Mission. This paper presents an improved approach of [3].

\section{ALGORITHM}

The phase unwrapping correction procedure combines global and local approaches. The idea is first to unwrap both interferograms separately using the Minimum Cost Flow (MCF) algorithm [4], second to compare the unwrapped phases to detect regions where they differ and finally to correct these regions with the help of the differential interferogram. This correction procedure has several levels of complexity. The processing is a part of the dual-baseline interferometric chain of the Integrated TanDEM-X Processor (ITP, [5]) which is further explained in [6].

\subsection{Available data and information}

The different data available from the ITP to achieve a reliable dual-baseline phase unwrapping (Figure 1) are:

- Two interferograms, one from the second coverage called master interferogram $I_{m}$ and one from the first coverage called slave interferogram $I_{s}$. They have the same

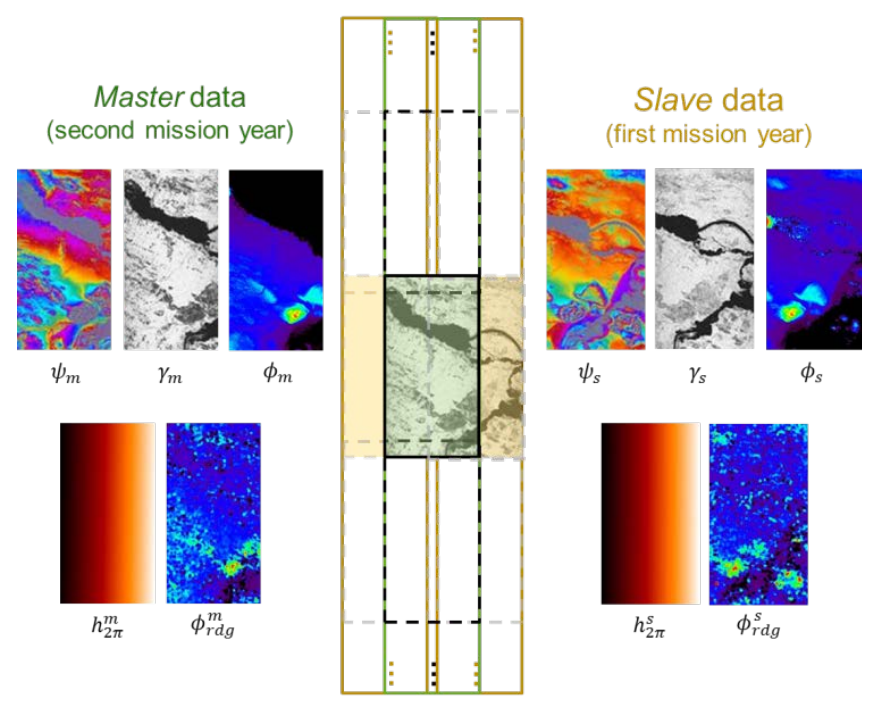

Figure 1: Available data for the dual-baseline phase unwrapping 
acquisition geometry and different baselines. The wrapped phases $\psi_{m}$ and $\psi_{s}$ are used.

- The coherences $\gamma_{m}$ and $\gamma_{s}$.

- The heights of ambiguity $h_{2 \pi}^{m}$ and $h_{2 \pi}^{s}$. Typical values are 30 to $35 \mathrm{~m} /$ cycle and 45 to $50 \mathrm{~m} /$ cycle giving a height of ambiguity ratio $\alpha$ of about 0.7 .

- Two stereo-radargrammetric phases $\phi_{r d g}^{m}$ and $\phi_{r d g}^{s}$. We refer to a radargrammetric phase as an absolute pseudophase. These are absolute measurements but have a coarse spatial resolution. They are obtained by converting the locally varying radargrammetric range offset estimates into phases. In the ITP, those mutual shifts between the two SAR images are estimated by patchwise signal cross-correlation [7, 8].

The following sections will detail the different steps of the algorithm.

\subsection{Phase unwrapping consistency check}

Two unwrapped phases can be compared by transforming both into heights via their height of ambiguity. The height difference is computed to check the phase unwrapping consistency and possible discrepant regions are identified. A detailed explanation of this procedure can be found in [6].

\subsection{Phase unwrapping errors correction}

The differential interferogram (height of ambiguity of about $100 \mathrm{~m} /$ cycle) is used to correct the phase unwrapping errors. Once it is unwrapped [6], the unwrapping correctness is checked and, if necessary, corrected using the stereoradargrammetric phase. This algorithm will be explained in section 3 .

Thereafter, the master unwrapped phase can be corrected. It is performed for all regions detected previously. Only the ambiguity band of the unwrapped phase is corrected, which makes the algorithm congruent (Figure 2).

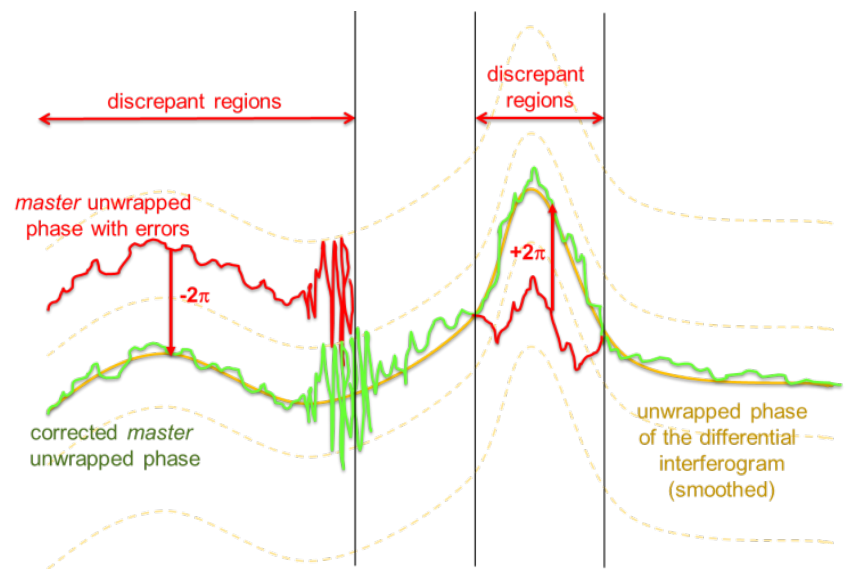

Figure 2: Principal of the phase unwrapping errors correction

\section{DIFFERENTIAL INTERFEROGRAM PROCESSING}

\subsection{The compatibility}

A local quality measure is needed for further masking and weighting during the processing. This new quality parameter, called compatibility, has been found empirically using the geometric mean of the coherences. It is defined by:

$$
\xi=\sqrt{\gamma_{d} \cdot \sqrt{\gamma_{m} \gamma_{s}}}
$$

where $\gamma_{m}, \gamma_{s}$ and $\gamma_{d}$ are respectively the coherences of the master, slave and differential interferograms.

Its characteristics are (Figure 3):

- when both master and slave interferograms are incoherent, the compatibility is low,

- the compatibility may be low although both interferograms are coherent: the terrain may have changed between the two acquisitions; therefore, the compatibility depends also on the differential interferogram coherence $\gamma_{d}$,

- persistent areas are highly compatible.
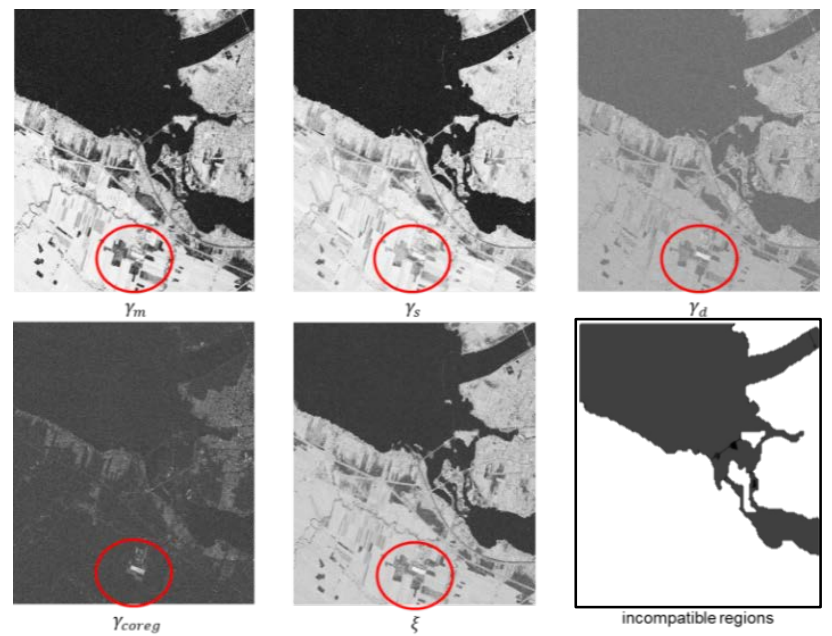

Figure 3: Coherences and compatibility images. It can be seen that the persistent areas are clearly visible (e.g. building at the bottom)

The compatibility is also used to control the master unwrapped phase correction. In fact, this phase does not have to be corrected with the differential interferogram when $I_{m}$ and $I_{s}$ are incompatible. The "correction" would even introduce some new errors.

\subsection{Differential interferogram correctness check}

This differential interferogram is easier to unwrap due to its higher height of ambiguity [6] but even then the unwrapped phase may be wrong. For that reason, its correctness is checked using the stereo-radargrammetric phase $\phi_{r d g}^{d}$. Nonetheless, we don't have $\phi_{r d g}^{d}$ itself but an equivalent of 
it is generated from the individual radargrammetric measures of both interferograms $\phi_{r d g}^{m}$ and $\phi_{r d g}^{s}$. Consequently, converting these two phases into height, two equivalent height maps are obtained which can be averaged to enhance the accuracy. $\phi_{r d g}^{d}$ is finally computed by scaling back this height map to the differential interferogram geometry in the following way:

$$
\phi_{r d g}^{d}=\frac{1}{h_{2 \pi}^{d}}\left(\frac{\phi_{r d g}^{m} h_{2 \pi}^{m}+\phi_{r d g}^{S} h_{2 \pi}^{s}}{2}\right)
$$

The correctness check is performed in a similar way as in the usual phase unwrapping quality check of the ITP [8]. First, the unwrapped phase is downsampled to the raster of $\phi_{r d g}^{d}$. Thereafter, both unwrapped and stereoradargrammetric phases are compared: if they do not agree, the histogram of the phase differences will exhibit several peaks. In contrast to the phase unwrapping consistency check described in [6], the correct ambiguity band can be retrieved directly from the histogram. Indeed, the stereoradargrammetric phase is absolute, so it does not require any phase unwrapping and thus, is error free apart maybe from a constant offset. Thereby, local maxima depict the unwrapping errors in the differential interferogram unwrapped phase. However, the correction has to be carried out carefully due to the low resolution of the stereoradargrammetric phase, especially at the border of the erroneous region.

\subsection{Analysis of the correction possibility}

In opposition to [8], the correction of the differential interferogram with the stereo-radargrammetric phase is possible for two reasons. On the one hand, this phase is obtained by averaging $\phi_{r d g}^{m}$ and $\phi_{r d g}^{s}$, so its accuracy is higher. On the other hand, the differential interferogram itself is corrected. Thus, the minimum height jump which has to be detected (corresponding to a phase unwrapping error) is much higher than the one in the master or slave interferograms. This is actually the main reason for a feasible correction as shown hereafter.

Given the standard deviation of the shift estimates from which the stereo-radargrammetric phase is derived [9], the standard deviation of the phase is:

$$
\sigma_{\phi_{r d g}}=\sqrt{\frac{3}{2 N}} \frac{\sqrt{1-\gamma^{2}}}{\pi \gamma} w_{r g}^{3 / 2} \frac{2 \pi f_{0}}{r s f}
$$

with $N$ the estimation window size (1024 in ITP), $\gamma$ the coherence, $w_{r g}$ the range oversampling factor (about 1.1 in ITP), $f_{0}$ the radar carrier frequency and $r s f$ the range sampling frequency. It is very important to notice that it does not depend on the baseline. then:

$$
\sigma_{\phi_{r d g}}^{d}=\frac{1}{2}\left|\frac{h_{2 \pi}^{m}}{h_{2 \pi}^{d}}\right| \sqrt{\sigma_{\phi_{r d g}}^{m}{ }^{2}+\left(\frac{h_{2 \pi}^{S}}{h_{2 \pi}^{m}}\right)^{2} \sigma_{\phi_{r d g}}^{s}{ }^{2}}
$$

We can replace the different height of ambiguity ratios by:

$$
\left|\frac{h_{2 \pi}^{m}}{h_{2 \pi}^{s}}\right|=\alpha \text { and }\left|\frac{h_{2 \pi}^{m}}{h_{2 \pi}^{d}}\right|=|1-\alpha| \text { with } \alpha>0
$$

Moreover, if we assume that $\gamma_{m}$ is equal to $\gamma_{s}, \sigma_{\phi_{r d g}}^{m}$ is thus equal to $\sigma_{\phi_{r d g}}^{s}$. We deduce the relation (6) between $\sigma_{\phi_{r d g}}^{d}$ and $\sigma_{\phi_{r d g}}^{m}$ which is plotted in Figure 4 .

$$
\frac{\sigma_{\phi_{r d g}}^{d}}{\sigma_{\phi_{r d g}}^{m}}=\frac{|1-\alpha|}{2} \sqrt{\left(1+\frac{1}{\alpha}\right)^{2}}
$$

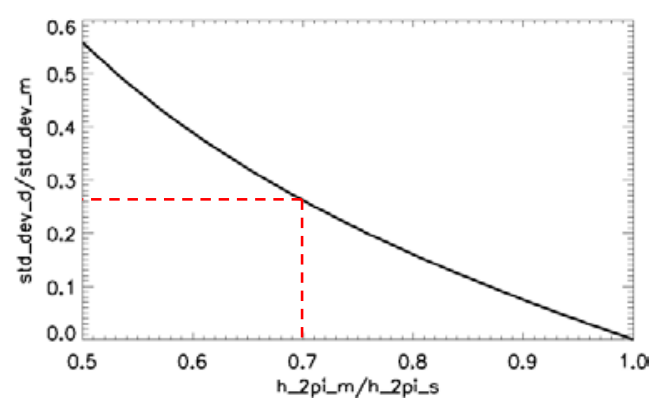

Figure 4: Ratio of the standard deviations $\sigma_{\phi_{r d g}}^{d} / \sigma_{\phi_{r d g}}^{m}$ versus the heights of ambiguity ratio $\alpha=h_{2 \pi}^{m} / h_{2 \pi}^{s}$ following equation (7)

For the typical height of ambiguity ratio $\alpha=0.7$, Figure 4 shows that $\sigma_{\phi_{r d g}}^{d}$ is almost 4 times smaller than $\sigma_{\phi_{r d g}}^{m}$. $\sigma_{\phi_{r d g}}^{m}$ being up to $2 \pi$ when the coherence is not low [8], $\sigma_{\phi_{r d g}}^{d}$ is then up to $0.5 \pi$ which is smaller than one ambiguity $(2 \pi)$. Thus, it allows us to detect the possible phase unwrapping errors in the unwrapped phase of the differential interferogram with these settings.

\subsection{Differential interferogram correction}

The phase unwrapping errors are detected on the downsampled version of the unwrapped phase. From every secondary peak of the phase differences histogram (Figure

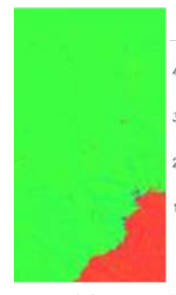

(a)
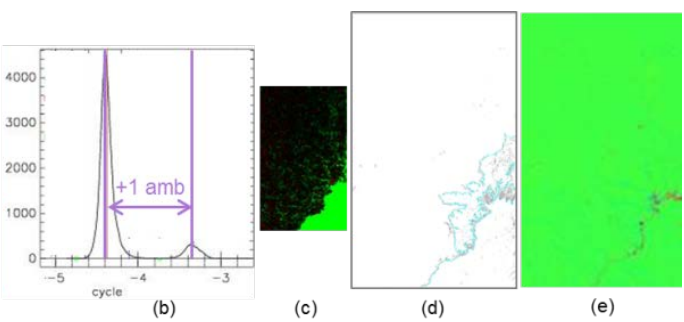

(d)

(e)

Figure 5: Different steps of the differential interferogram correction (a) difference to SRTM phase before correction (b) histogram of the difference with the stereo-radargrammetric phase (c) detected phase unwrapping errors (d) boundary mask (e) difference to SRTM phase after correction 
5.b), a region to be corrected by a certain ambiguity band is formed. A mask of ambiguity bands to be corrected is thereby generated (Figure 5.c). However, to be able to correct the unwrapped phase, it is necessary to go back to the full interferogram resolution. The critical step is to find the exact boundaries of these regions in the full resolution. The boundaries (Figure 5.d) are a combination of the boundary of the discrepancy map calculated in 2.2. and the incompatible regions (Figure 3). The regions are then clearly delimited and the correction is straightforward. Figure 5.a and Figure 5.e are a comparison with the SRTM phase: the green part shows agreement in terms of ambiguity bands.

\section{RESULTS}

An example of a problematic area is Lesotho, a country in South Africa. A part of the border between Lesotho and South Africa is a natural wall of more than 500 meters high. Phase unwrapping is very challenging due to the abrupt terrain changes especially with the TanDEM-X height of ambiguities. Figure 6 exhibits the relevant images from the different processing steps and the resulting corrections. As a visual check the master unwrapped phase is compared to the phase simulated from a SRTM DEM before (Figure 6.a) and after (Figure 6.e) the phase unwrapping errors correction. The green color exhibits an agreement in terms of ambiguity bands. Figure 7 shows a raw DEM delivered by the ITP with some profiles demonstrating the corrections.

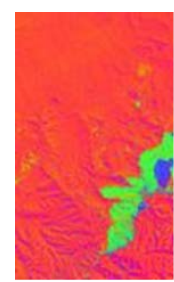

(a)

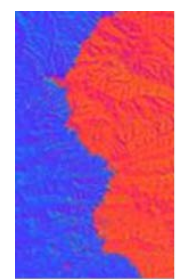

(a)

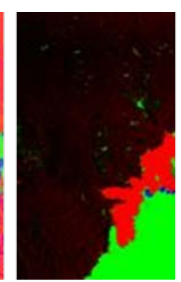

(b)

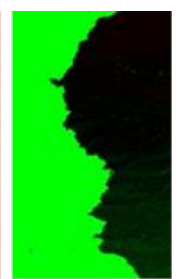

(b)

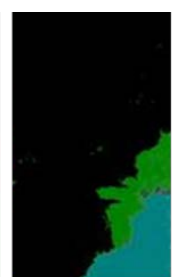

(c)

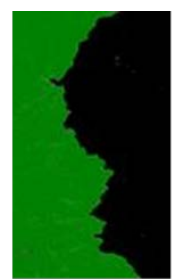

(c)

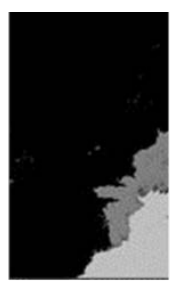

(d)

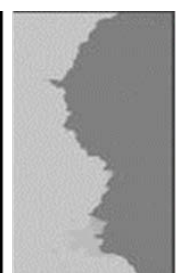

(d)

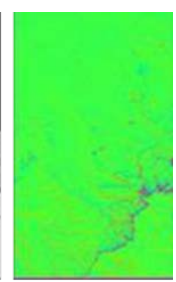

(e)

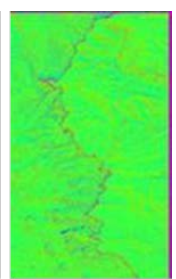

(e)
Figure 6: Images of the relevant processing steps of the dualbaseline phase unwrapping: (a) comparison with the SRTM phase before the dual-baseline correction (b) height discrepancies (c) mask of regions to be corrected (d) corrected ambiguity bands (e) comparison with the SRTM phase after the correction

\section{CONCLUSION}

A robust phase unwrapping algorithm has been developed using the advantages of the differential interferogram: it is easier to unwrap correctly and the possible unwrapping errors can be corrected using the stereo-radargrammetric phase. During the correction steps, only the ambiguity bands are changed, which makes the algorithm congruent.

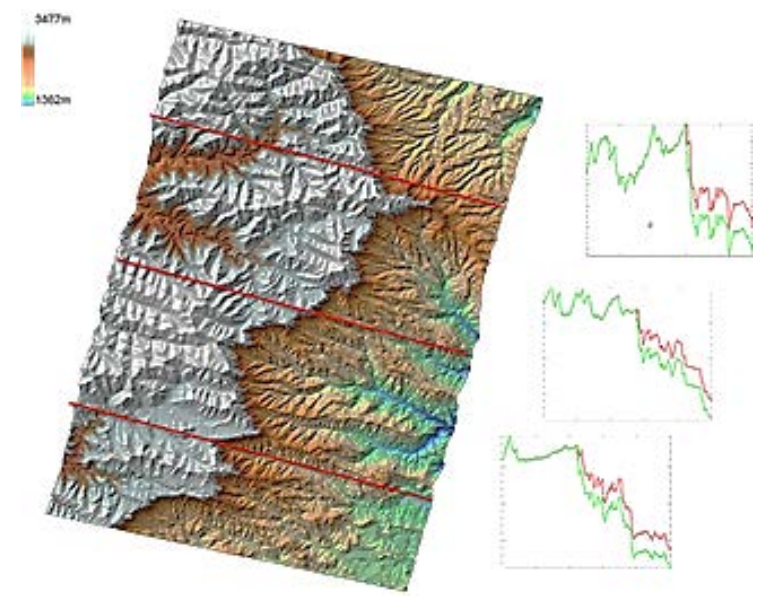

Figure 7: Raw DEM with profiles demonstrating the correction

\section{REFERENCES}

[1] G. Krieger, A. Moreira, H. Fiedler, I. Hajnsek, M. Werner, M. Younis, and M. Zink, "TanDEM-X: A satellite formation for High-Resolution SAR Interferometry", IEEE TGARS, vol. 45, No 11. November 2007

[2] M. Lachaise, R. Bamler, and F. Rodriguez-Gonzalez, "Multi-baseline gradient ambiguity resolution to support Minimum Cost Flow Phase Unwrapping”, Proc. IGARSS 2010, Honolulu, 2010

[3] M. Lachaise, T. Fritz, and M. Eineder, "A new dual baseline phase unwrapping algorithm for the TanDEM-X Mission”, Proc. EUSAR 2008, Friedrichshafen, 2008

[4] M. Eineder, M. Hubig, and B. Milcke, "Unwrapping large interferograms using the minimum cost flow algorithm", Proc. IGARSS 1998, Seattle, 1998

[5] T. Fritz, C. Rossi, N. Yague-Martinez, F. RodriguezGonzalez, M. Lachaise, and H. Breit, "Interferometric processing of TanDEM-X data”, Proc. IGARSS 2011, Vancouver, 2011

[6] M. Lachaise, U. Balss, T. Fritz, and H. Breit, "The dualbaseline interferometric processing chain for the TanDEM-X mission”, to be published in Proc. IGARSS 2012, Munich, 2012

[7] N. Yague-Martinez, M. Eineder, H. Breit, T. Fritz, and R. Brcic, "TanDEM-X Mission: SAR Image Coregistration Aspects”, Proc. EUSAR 2010

[8] C. Rossi, F. Rodriguez Gonzalez, T. Fritz, N. Yague Martinez, and M. Eineder, "Calibrated TanDEM-X Raw DEM Generation”, ISPRS J. Photogramm. Remote Sens., under review, 2012

[9] Bamler, R., and Eineder, M., “Accuracy of Differential Shift Estimation by Correlation and Split Bandwidth Interferometry for Wideband and Delta-k SAR Systems”, IEEE GSRSL, vol. 2(2), pp. 151-155, 2005. 